\title{
AVAILABILITY Modelling OF RECONFIGURABLE MANUFACTURING SySTEM
}

\author{
GuPTA, A.; JAIN, P. K. \& KUMAR, D.
}

Abstract: In this chapter, the stochastic model is developed to asses and to analyze the availability of reconfigurable manufacturing system capable of producing multiple-parts. Availability, as performance measure, reflects the ability of a system to satisfy demand requirements at any time. The evaluation of availability of a system is influenced by the availability and arrangement of its individual components. A reconfigurable manufacturing system (RMS) composed of reconfigurable machines is designed for cost-effective response to changes in production requirement through reconfiguration. Each reconfigurable machine has several performance states. Accordingly, the system is having a finite number of performance levels and belongs to the category of Multi-State Systems (MSS). The Universal Generating Function $(U G F)$ technique is fast and efficient in evaluating the availability of MSS. The present study contains the description of the UGF, its modification, capability and application along with the some guide lines for the future research with an objective to create the interest of research community to mature the approach.

Key words: availability, reconfigurable manufacturing system, universal generating function, multi state system
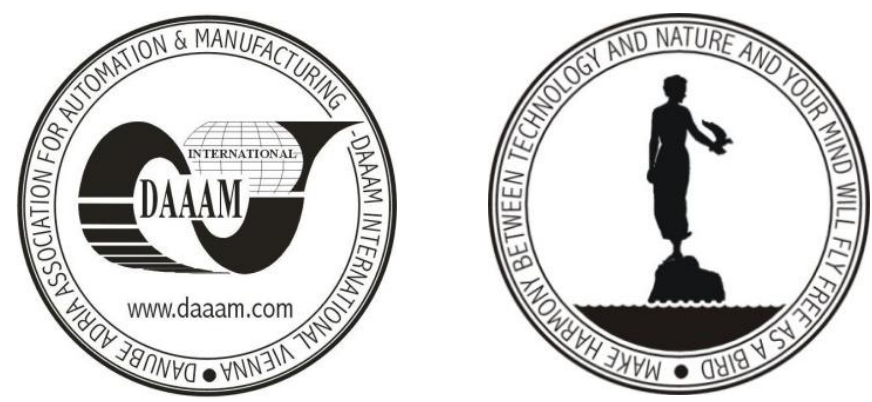

Authors' data: Gupta, A[shutosh]; Jain, P[ramod] K[umar]; Kumar, D[inesh], Mechanical \& Industrial Engineering Department, Indian Institute of Technology Roorkee, India, ashuaec72@gmail.com,_pjainfme@iitr.ernet.in, dinesfme@iitr.ernet.in

This Publication has to be referred as: Ashutosh, A[shutosh]; Jain, P[ramod] K[umar] \& Kumar, D[inesh] (2012). Availability Modelling of Reconfigurable Manufacturing System, Chapter 21 in DAAAM International Scientific Book 2012, pp. 241-254, B. Katalinic (Ed.), Published by DAAAM International, ISBN 978-3901509-86-5, ISSN 1726-9687, Vienna, Austria

DOI: $10.2507 /$ daaam.scibook.2012.21 
Gupta, A.; Jain, P. K. \& Kumar, D.: Availability Modelling of Reconfigurable Man...

\section{Introduction}

Manufacturing companies in the 21 st century face increasingly frequent and unpredictable market changes driven by global competition, including the rapid introduction of new products and constantly varying product demand. To remain competitive, companies must design manufacturing systems that not only produce high-quality products at low cost, but also allow for rapid response to market changes and consumer needs. Reconfigurability is a novel engineering technology that facilitates cost effectiveness and rapid responses to market and product changes. A cost effective response to market changes requires a new manufacturing approach. Such an approach not only must combine the high throughput of a DML with the flexibility of FMS, but also be capable of responding to market changes by adapting the manufacturing system and its elements quickly and efficiently. These capabilities are encompassed in reconfigurable manufacturing systems (RMS), whose capacity and functionality can be changed exactly when needed (Koren et al., 1999). Customization, scalability and convertibility (Koren et al., 2003) are critical reconfiguration characteristics. Implementing RMS characteristics and principles in the system design leads to achieving the ultimate goal to create a "living factory" that can rapidly adjust its production capacity while maintaining high levels of quality from one part to the next. This adaptability guarantees a high long-term profit-to-cost-ratio and rapid return on investment of reconfigurable manufacturing systems. Lokesh and Jain (2011) developed a three-phased methodology to decide RMS configuration in the desired period considering various features such as multiproduct line, number of stages, selection of machine type, machine configuration, number of same type parallel machine in a stage, machine selection for all stages, allocation of stage functionality blocks in each stage, etc., and various constraints and performance measures satisfaction, but existing configuration consideration is also important to select RMS configuration in the desired period. A system's configuration facilitates or impedes its productivity, responsiveness, convertibility and scalability, and also impacts its daily operations. Multi-stage manufacturing system allows for several operational configurations, depending on how the machines are arranged in the stages and how they are connected via the material handling system. In the general case the total number of configurations for $\mathrm{N}$ machines is huge. The number of possible configurations increases exponentially with the number of machines. However, the number of possible RMS configurations is much smaller. System configurations are classified either as symmetrical or as asymmetrical, based on whether a symmetric axis is drawn along the configuration. Asymmetric configurations add immense complexity and are not viable in real manufacturing lines, because of non-identical flow-paths for the parts. They therefore need several process plans and corresponding setups. Different process plans and corresponding flow-paths increase part quality problems and make quality error detection more complicated. It is more likely that in a real manufacturing context, only symmetric configurations are considered; these are always single process configurations with identical machines in each stage. The type of systems configurations has an important impact on their performance. Different types of manufacturing systems performance 
measures are reviewed (Hon, 2005). The performance of the system configurations depends upon how much the system is available to meet the required demand of the products. This ability of a system configuration to satisfy production demand requires its availability evaluation. The evaluation of availability of a system configurations are influenced by the availability and arrangement of its individual components. ElMaraghy, H. et al. (2005) introduced the notion of availability as a functional requirement and used it to compare manufacturing systems complexity. Manufacturing system specifically reconfigurable manufacturing systems are typically composed of group of reconfigurable machines/stations in a specific arrangement. These individual machines/stations have either identical or different performance levels (production rates). In addition, each of these individual machines/stations has several performance states (e.g. operating, idle, down or under repair). Accordingly, reconfigurable manufacturing systems have a finite number of performance levels. Therefore, it belongs to the category of Multi-State Systems (MSS). A binary system is the simplest case of a MSS having two distinguished states (perfect functioning and complete failure). There are others situations in which a system are to be considered to be a MSS. Any system consisting of different units that have a cumulative effect on the entire system performance is also to be considered as a MSS. Indeed, the performance rate of such a system depends on the availability of its units, as the different numbers of the available units provide different levels of the task performance.

The MSS was introduced in the middle of the 1970s in (Murchland, 1975). In this works, the basic concepts of MSS reliability were formulated; the system structure function was defined for coherent MSS, and its properties were investigated. The reliability importance was extended to MSS (Griffith, 1980). The concept of equivalent behavior was introduced (Garriba et al., 1980) to provide a comprehensive description of states and state transitions in the MSS and its components. Pouret et al. (1999) was developed a method for the two-sided estimation of MSS unavailability. The method was based on the binary model, which can be assessed with the usual tools. An asymptotic approach to the MSS reliability evaluation was presented (Kolowrocki, 2000). In real-world problems of MSS reliability analysis, the great number of system states that need to be evaluated makes it difficult to use traditional techniques in various optimization problems. Traditional techniques for assessment of MSS reliability (availability) include Boolean-based methods, such as minimal cut sets (Aven, 1985) and fault tree technique (Vesely et al., 1981) can be represented by the Monte-Carlo simulation for the reliability assessment, the main disadvantages of these approaches are the time and expenses involved in the development and execution of the model. The approach based on the extension of Boolean models is historically the first method that was developed and applied for the MSS availability evaluation. It is based on the natural expansion of the Boolean methods to the MSS. The main difficulties in such analysis are the 'dimension damnation' since each system element can have many different states (not only two states as existed in the binary-state system). This makes the Boolean approach overworked and time consuming. 
Stochastic-based methods, mainly Markov and semi-Markov processes (Limnios \& Oprisan, 2001) are widely used for the MSS reliability (availability) analysis are more universal. In fact, this approach was successfully used for the assessment of multi-state power systems and some types of communication systems even before MSS was theoretically defined. The stochastic process method can be applied only to relatively small MSS because the number of system states increases dramatically with the increase in the number of system elements. The computational burden is the crucial factor when one solves optimization problems. These techniques are inefficient and extremely time consuming if applied to large MSS because of the high number of system states (Lisniansk \& Levitin, 2003). In contrast, the Universal Generating Function (UGF) technique is fast enough to be used in these problems. This technique allows to find the entire MSS steady-state performance distribution (PD) based on the steady-state PD of its elements by using a fast algebraic procedure. In this analyst use the same recursive procedures for MSS with a different physical nature of performance and different types of element interaction. The u-function is extension of widely known ordinary moment generating function. The essential difference between the ordinary and UGF is that the latter allows one to evaluate probabilistic distributions of overall performance.

The Universal Generating Function technique was first introduced by Ushakov (1986), proved to be efficient in evaluating the availability of large MSS (Levitin \& Lisnianski, 1999). However, it has never been applied to reconfigurable manufacturing systems. In addition, the application of UGF to MSS to date is limited to the evaluation of systems with multiple type of output performance. The application of UGF in assessing the availability of reconfigurable manufacturing system is developed in this study. A modification of the original method to generalize its use and extend it to RMS with multiple types of output performance is introduced. This enables the application of the UGF technique to reconfigurable manufacturing systems capable of producing multiple part types simultaneously. This technique allows finding the entire system availability based on the steady-state performance distribution of its elements by using a fast algebraic procedure.

\section{Universal Generating Function (UGF):}

\subsection{Brief Description:}

The UGF (u-function) technique was proved to be very effective for the reliability evaluation of different types of MSS (Lisniansk \& Levitin, 2003). In particular, the UGF enables to assess availability of multi-state systems. UGF allows evaluating probabilistic distributions of overall performance for wide range of systems characterized by different topology, different nature of interaction among system elements and different physical nature of element's performance measures. This is done by introducing different composition operators over the UGF. The main assumptions when using the UGF technique was that the system elements are mutually statistically independent. 
The UGF of the distribution of a discrete random variable $\mathrm{X}$ (can be any stochastic performance level), which can have $K$ values $\left(a_{1}, a_{2}, \ldots, a_{K}\right)$, is the function $U(Z)$ defined for all real numbers $Z$ by:

$$
U(Z)=\sum_{i=1}^{K} p_{i} Z^{a_{i}}
$$

where $p_{i}$ is the probability that the random variable $X$ under consideration takes the value $a_{i}$, and $Z$ is the argument of the generating function.

Consider systems described as reducible structures, i.e., structures that can be represented as compositions of serial and parallel connections of a group of components (e.g. manufacturing system configurations). A characteristic property of such systems is that each of them can be reduced to a single equivalent component by means of a finite number of composition operators. Composition operators are used to obtain the overall UGF of these systems by applying simple algebraic operations to the UGF of their components. Steady-state availability of a repairable system, as a performance measure, is the probability that the system is, on average, performing satisfactorily over a reasonable period of time (Lewis, 1987). To obtain steady state probability distributions of the different states of a multi-state system based on the probability distributions of the states of its individual components, the composition operator $\phi$ is defined by:

$$
\phi\left[U_{i}(Z), U_{j}(Z)\right]=\sum_{\text {all i ill } \mathrm{j}} \sum_{i} p_{j} Z^{f\left(a_{i}, a_{j}\right)}
$$

The $f\left(\mathrm{a}_{\mathrm{i}}, \mathrm{a}_{\mathrm{j}}\right)$ is defined according to the physical nature of the multi-state system performance and the interactions between its components. It expresses the entire performance level of a subsystem consisting of two components connected in parallel or in series in terms of the performance levels of its individual components.

Let $\pi$ be the composition operator corresponding to a parallel connection of components and $\sigma$ is the composition operator for a series connection. Composition operator's $\pi$ and $\sigma$ are special cases of $\phi$. For MSS that uses capacity of its components as its performance level (e.g. production rates in our case), the two operators, $\pi$ and $\sigma$ are defined as follows:

- The system total performance level is the addition of the performance levels of all components in parallel arrangement. Accordingly, the $\pi$ operator is the product of the individual UGF of system components and $\otimes$ is the composition operator over U-function for the elements connected in parallel or series:

$$
\pi\left[U_{i}(Z), U_{j}(Z)\right]=\sum_{\text {all } \mathrm{i}} p_{i} Z^{a_{i}} \underset{\text { par all } j}{\otimes} \sum_{j} Z^{a_{j}}=\sum_{\text {all ial j j }} \sum_{i} p_{j} Z^{\operatorname{par}\left(a_{i}, a_{j}\right)}
$$

- The system total performance level is the minimum of the performance levels of all components in serial arrangement. Accordingly, the $\sigma$ operator is applied 
to choose the minimum performance level which corresponds to the bottleneck component:

$$
\sigma\left[U_{i}(Z), U_{j}(Z)\right]=\sum_{\text {all } \mathrm{i}} p_{i} Z^{a_{i}} \underset{\text { ser }{ }_{\text {all }} j}{ } p_{j} Z^{a_{j}}=\sum_{\text {all ial } j} \sum_{i} p_{j} Z^{\text {ser }\left(a_{i}, a_{j}\right)}
$$

Evidently, a successive application of the composition operators, $\pi$ and $\sigma$, reduces any reducible structure to an equivalent component. Consequently, the UGF of the entire multi state system is obtained in the form:

$$
U_{\text {sssem }}(Z)=\sum_{\text {all } i} p_{i} Z^{a_{i}}
$$

\subsection{UGF Modification}

A modification to the UGF is developed to consider systems with multiple independent types of output performance that collectively affect the assessment of the performance measure of the system. These output performance types (e.g. production rates for multiple part types in RMS) is to be expressed, in such a case, by a vector, the length of which is the number of these types rather than a single variable. Accordingly, the UGF expressed in (1) is now be replaced by:

$$
U(Z)=\sum_{i=1}^{K} p_{i} Z^{G_{i}}
$$

where is $\mathrm{G}_{\mathrm{i}}$ the output performance types vector.

In MSS of this type, the total performance rate of a pair of elements connected in parallel is equal to the sum of the performance rates of the individual elements. When the elements are connected in series, the element with the lowest performance rate becomes the bottleneck of the subsystem. Therefore, for a pair of elements connected in series the performance rate of the subsystem is equal to the minimum of the performance rates of the individual elements. When applying the composition operators $\pi$ and $\sigma$, expressed in (3) and (4), the summation/comparison are now vector operations applied to corresponding elements of vectors $G_{i}$ and $G_{j}$ The resultant vector is the same size and includes the performance level corresponding to each type of output performance. The modified operators are now expressed as follows:

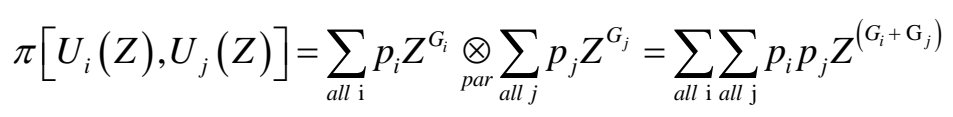

And

$$
\sigma\left[U_{i}(Z), U_{j}(Z)\right]=\sum_{\text {all } \mathrm{i}} p_{i} Z^{G_{i}} \underset{\text { ser }}{\otimes} \sum_{\text {all } j} p_{j} Z^{G_{j}}=\sum_{\text {all } \mathrm{i} \text { all } j} p_{i} p_{j} Z^{\min \left(G_{i}, G_{j}\right)}
$$


By applying the modified composition operators, the UGF of the entire multi state system is now obtained in the form:

$$
U_{\text {system }}(Z)=\sum_{\text {all } i} p_{i} Z^{G_{i}}
$$

\section{Application to Reconfigurable Manufacturing System}

\subsection{Availability of RMS}

Consider the reconfigurable manufacturing system requires the $\mathrm{N}$ machines for processing the parts family. Figure 1 show the typical part family in which part two parts variant are to be manufactured in reconfigurable environment. The number of machines required depends upon the parts demand and the total processing time for the part and the time available in the particular day/ shift. This yielded system designer, the minimum number of the machines needed to meet the required demand. The next step is to arrange these machines in the one of the possible configurations to take the advantage as much as possible in terms of productivity and cost. In general, the number of possible configurations for the $\mathrm{N}$ machines is huge but the number of possible RMS configurations is much smaller as indicated in the Table 1. The basic equation for calculating the number of possible configurations is as follows:

$$
K=\sum_{i=1}^{N}\left(\frac{N-1}{m-1}\right)=2^{N-1}
$$

where, $K$ is the number of possible configurations with $N$ machines arranged in the exactly $m$ stages. For example, for $N=6$ machines arranged in up to 6 stages, eq. (10) yields $K=32$ configurations, and if arranged in exactly 3 stages yield $K=10$ configurations. The mathematical results of the equation (10) for any $N$ and be arranged in the triangular format is called Pascal Triangle as shown in the Table 1. This triangle allowed system designer to immediately calculate and visualize the number of the possible RMS configurations for $\mathrm{N}$ machines arranged in the $\mathrm{m}$ stages.

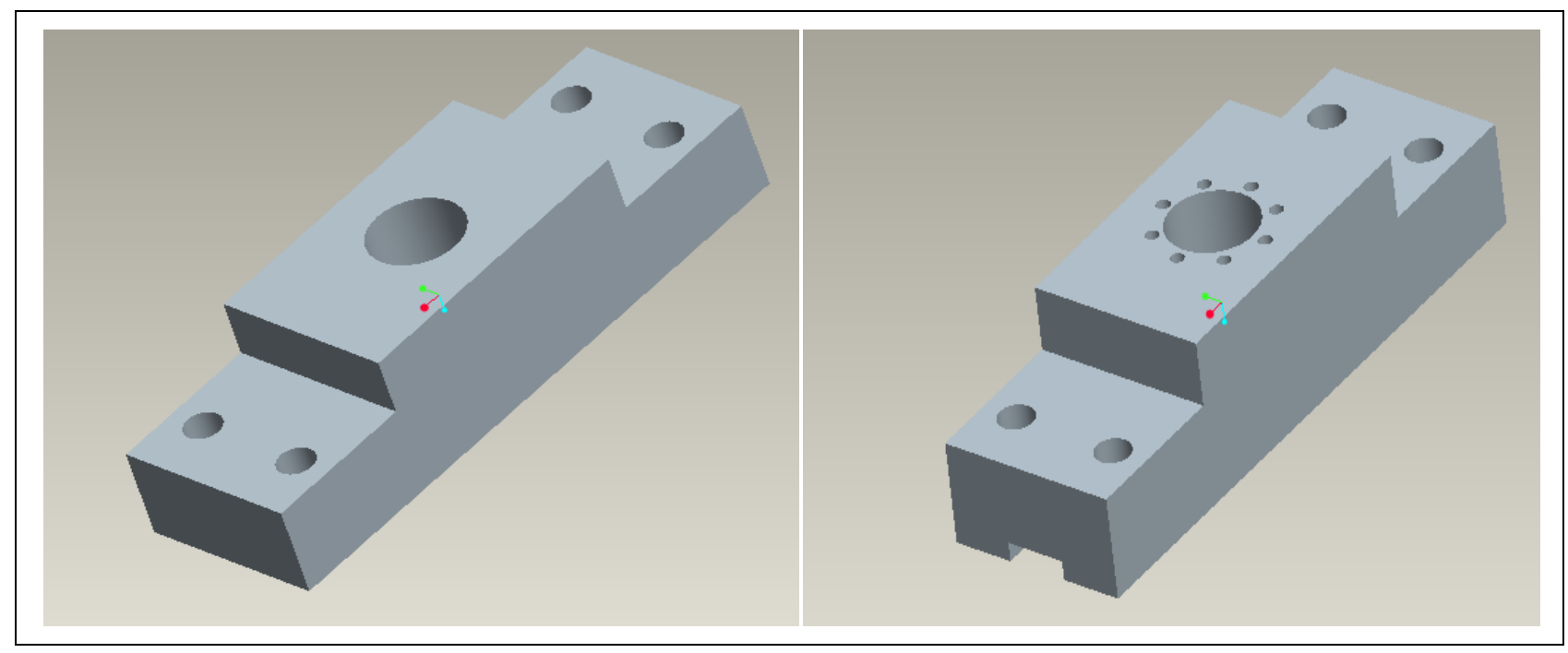

Fig. 1. A typical part and its variant 


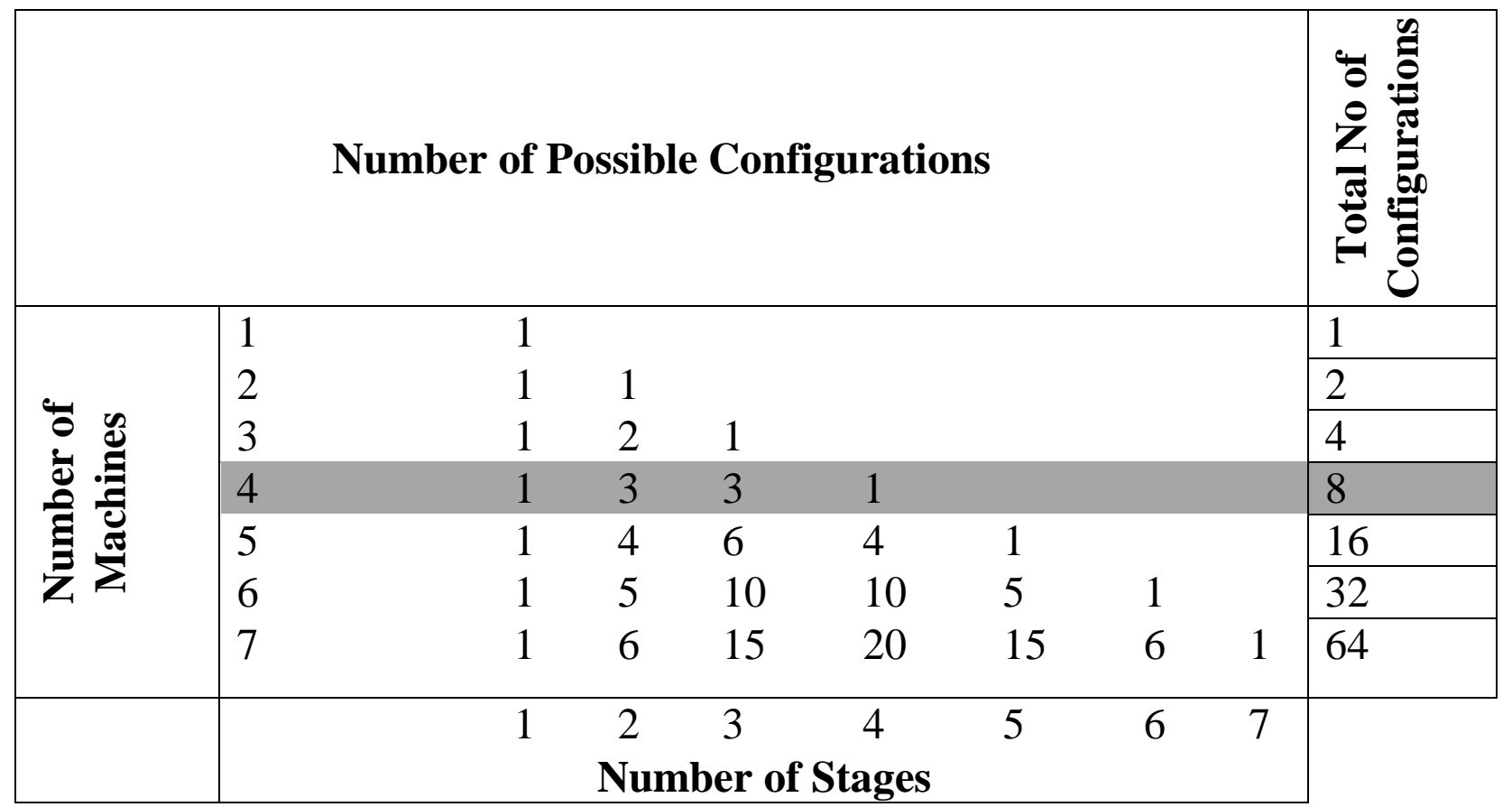

Tab. 1. Pascal Triangle for Calculating the Number of possible configurations

Figure 2 shows a typical configuration structure used in the reconfigurable manufacturing system which is the flow line that allow the parallel of machines/stations in different production stages. The presence of multiple parallel machines/stations per stage reduces the effect of breakdown of any of the machines thus the use of buffers is not always essential. This typical reconfigurable structure is capable of producing the multiple parts.

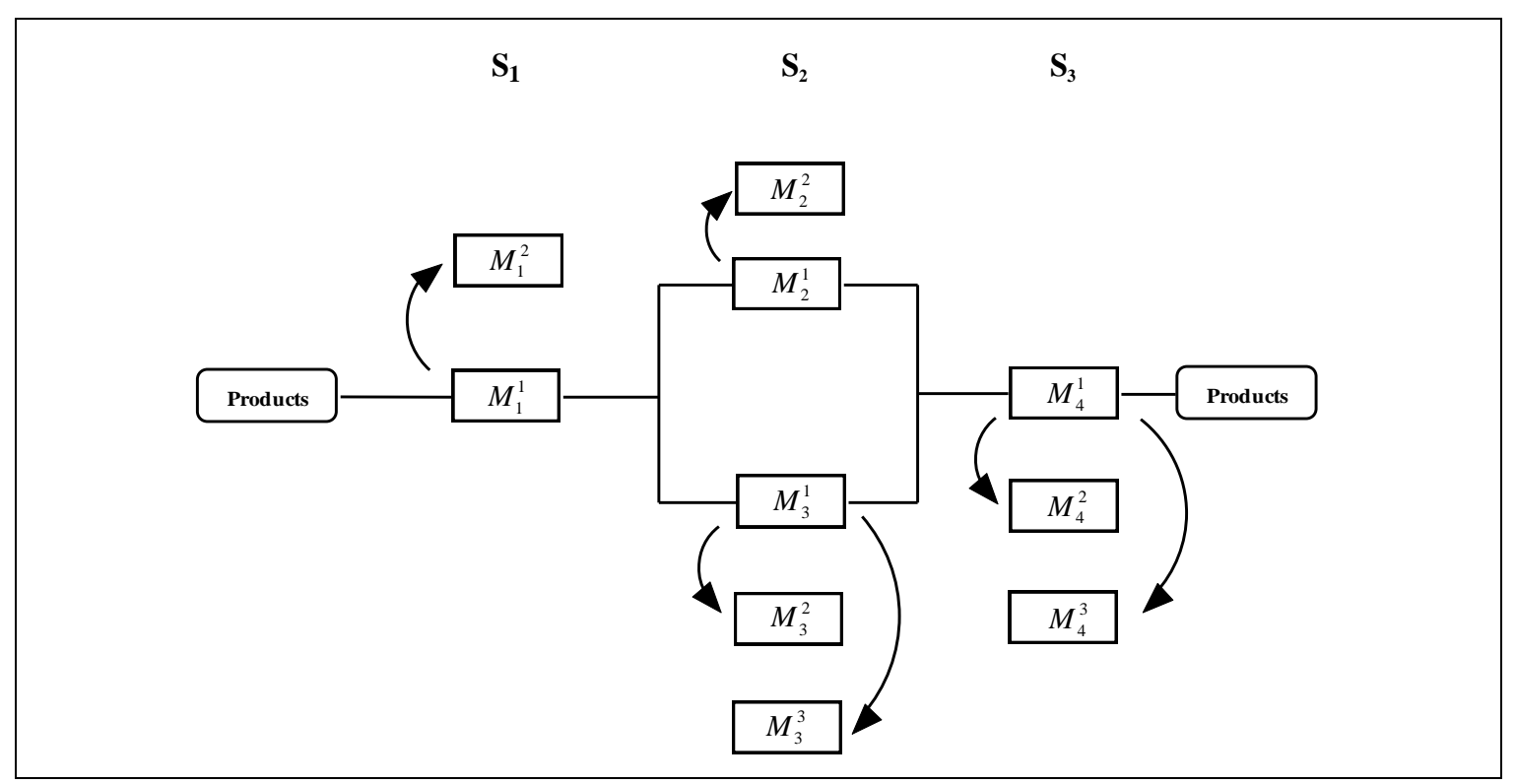

Fig. 2. A Typical Reconfigurable Manufacturing System

In Figure 2, $S_{i}$ stands for $\mathrm{i}^{\text {th }}$ stage, $M_{i}^{j}$ for $\mathrm{i}^{\text {th }}$ machine/station in $\mathrm{j}^{\text {th }}$ configuration. Here The reconfigurable manufacturing system exemplified in Figure 2 is a MSMS that falls under the category of reducible structures. Accordingly, the application of UGF in evaluating its availability is justified. 
In the context of RMS, the system availability, defined in Section 2.1, is considered a measure of the ability of the system to satisfy the demand requirements. To evaluate the steady-state availability of the system, the availability of its individual components (machines/stations) and their individual performance levels for different types of output performance (i.e. production rates corresponding to multiple parts types being produced) is considered.

\subsection{Application of $U G F$}

Consider the steady-state availability of each individual machine/station $\mathrm{j}$ with two possible states (operating or failed) to be $R_{\mathrm{j}}$. The performance level of this machine/station is a vector of all output performance types (production rates corresponding to each part type). This performance level can either be 0 (when failed) with probability of occurrence of $\left(1-R_{\mathrm{j}}\right)$ or $\mathrm{PR}_{\mathrm{j}}$ (when operating) with probability of occurrence of $R_{\mathrm{j}}$ where $\mathrm{PR}_{\mathrm{j}}$ is a vector of nominal production rates corresponding to each part type. In such case, the polynomial UGF in eq. (6) has only two terms as follows:

$$
U_{j}(Z)=\left(1-R_{j}\right) Z^{0}+R_{j} Z^{P R_{j}}
$$

The UGF of the entire system is obtained through successive applications of the composition operators as described in eq. (7) and eq. (8). It represents all the possible states of the system by relating the probability of each system state to the expected performance of the system in that state. The system availability is the probability that the system is in one of those states in which the system production rates satisfy the target demand requirements, which is the summation of the probabilities of occurrence of those states.

\section{Numerical illustration}

For illustrating the above approach, a reconfigurable manufacturing system that produces multiple part types simultaneously is considered to explain the application of the modified UGF technique in evaluating system availability and its computational merits. The said system is capable to produce the typical part and its variant simultaneously as shown in the figure 1. Four reconfigurable machines are required to complete all the desired operations for producing the typical part type. The steady state availability of the reconfigurable machines is calculated by using the equations (12) and (13) and presented in Table 2.

$$
\begin{aligned}
& \mathrm{MTTF}_{\mathrm{M}}=\mathrm{MTTF}_{\mathrm{C}} / \text { No. of Components } \\
& \text { Machine Availability }(\mathrm{R})=\frac{\mathrm{MTTF}_{\mathrm{M}}}{\left(\mathrm{MTTF}_{\mathrm{M}}+\mathrm{MTTR}_{\mathrm{M}}\right)}
\end{aligned}
$$

where, MTTF is the mean time to failure and MTTR is the mean time to repair for components and the machine. The MTTF and MTTR for each machine are taken hypnotically but for practical application, it is available to the system designer a 
priori. The demand for two parts is assumed 120 and 180 for initial period and system is capable to meet the additional demand in subsequent reconfiguration.

In the example problem, once the number of machines is taken to complete all operations, the next step of the system designer/analyst is to calculate the number of total possible symmetrical configuration using the equation 10 or directly it is taken according to the table 1 . The number of possible RMS configurations for our problem is eight, are further classified in terms of number of stages as highlighted in table 1. These symmetrical configurations do require simple material handling system for loading /unloading the station as well as they provide bidirectional material movement. Also, variable process plan can easily adoptable for these configurations. The all possible symmetrical configurations for the example problem are presented in Figure 3.

\begin{tabular}{|c|c|c|c|c|c|c|}
\hline & & & & & \\
\hline & & & & & \\
\hline $\mathbf{M}_{1}^{1}$ & 10 & 1000 & 20 & 100 & 20 & 0.833 \\
\hline $\mathbf{M}_{2}^{1}$ & 10 & 1000 & 20 & 100 & 20 & 0.833 \\
\hline $\mathbf{M}_{3}^{1}$ & 10 & 1000 & 20 & 100 & 20 & 0.833 \\
\hline $\mathbf{M}_{4}^{1}$ & 10 & 1000 & 20 & 100 & 20 & 0.833 \\
\hline $\mathbf{M}_{2}^{2}$ & 15 & 1000 & 20 & 66.67 & 20 & 0.769 \\
\hline $\mathbf{M}_{2}^{2}$ & 15 & 1000 & 20 & 66.67 & 20 & 0.769 \\
\hline $\mathbf{M}_{3}^{2}$ & 20 & 1000 & 20 & 50 & 20 & 0.714 \\
\hline $\mathbf{M}_{3}^{2}$ & 20 & 1000 & 20 & 50 & 20 & 0.714 \\
\hline $\mathbf{M}_{4}^{2}$ & 30 & 1000 & 20 & 33.33 & 20 & 0.625 \\
\hline $\mathbf{M}_{4}^{3}$ & 30 & 1000 & 20 & 33.33 & 20 & 0.625 \\
\hline
\end{tabular}

Tab. 2. Steady state availability

In Figure 3 configuration A is pure serial and of the four stages. Configuration $\mathrm{H}$ is of pure parallel and of single stage. The remaining configurations are hybrid and of two and three stages respectively. These possible configurations gave the different availability value in terms of their performance. In order to obtain the UGF representing the system configuration availability performance, we first defined the U-function for each element (machine) such as $U_{1}(Z), U_{2}(Z), U_{3}(Z)$ and $U_{4}(Z)$ in accordance with the eq.(11) for machines $M_{1}^{1}, M_{2}^{1}, M_{3}^{1}$ and $M_{4}^{1}$ respectively.

$$
\left.U_{1}(Z)=(1-0.833) Z^{[0]}+0.833 Z^{[1200}\right]
$$


Similarly, we calculated the $\mathrm{U}_{2}(\mathrm{Z}), \mathrm{U}_{3}(\mathrm{Z})$ and $\mathrm{U}_{4}(\mathrm{Z})$ which is come out equal to the $U_{1}(Z)$. This is because the individual machine availability and their production rates are taken equal in the example problem.

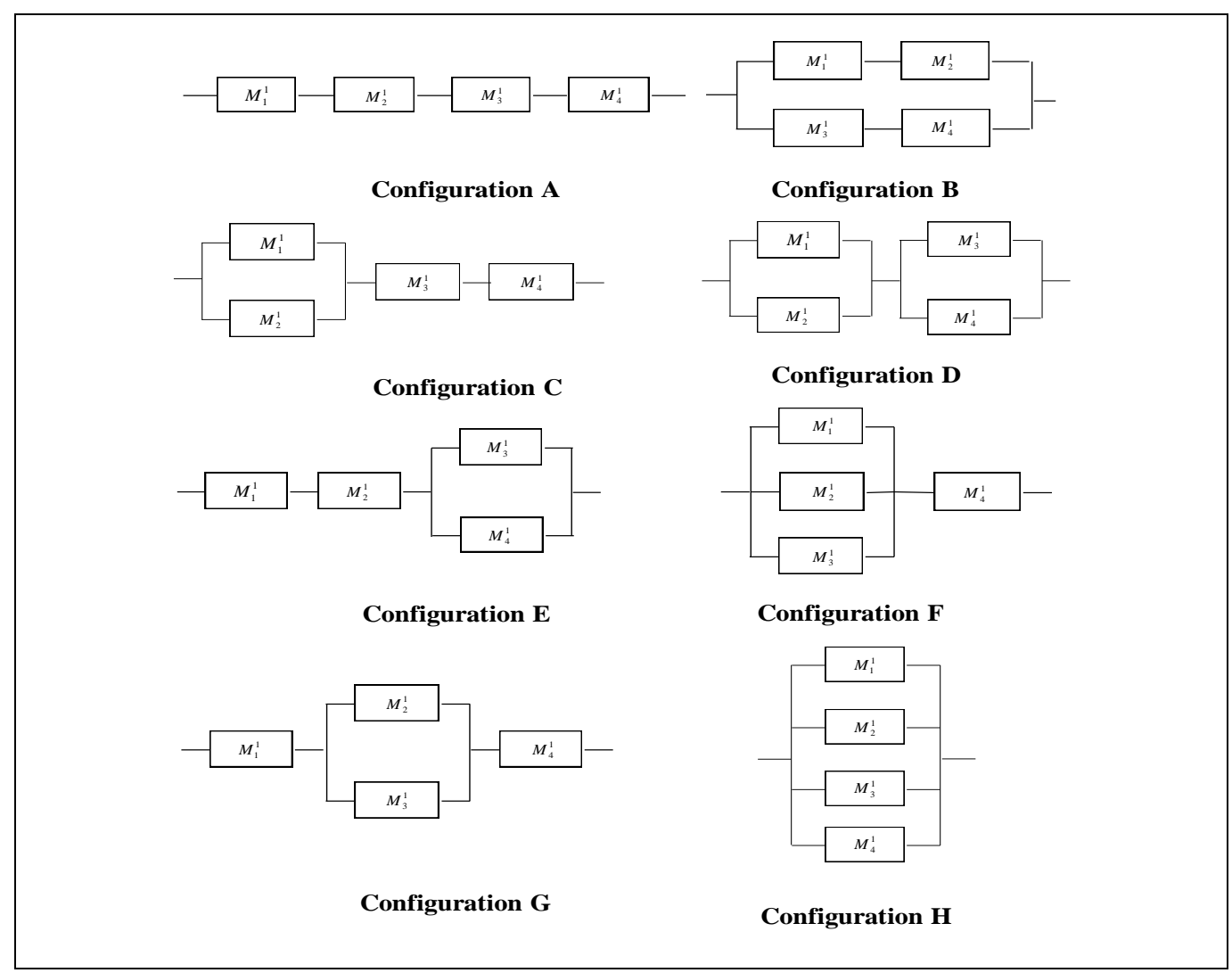

Fig. 3. Different symmetrical system configurations of four RMT

Once the U- function for the individual machines (elements) are determined then obtain the U-function for the pair of mutually independent elements connected in a series or in parallel and replace this pair with an equivalent elements with Ufunction obtained by the composition operators using eq. (7) and eq.(8). If the system configuration contains more than one pair of element then repeat the above procedure.

Lets us take the configuration D for sample calculations for the illustration. This particular configuration is having two stages. Each stage is having two RMT which are parallel and then connected in series. First, the U-function for the pair of elements connected in parallel (stage1) is calculated as follows:

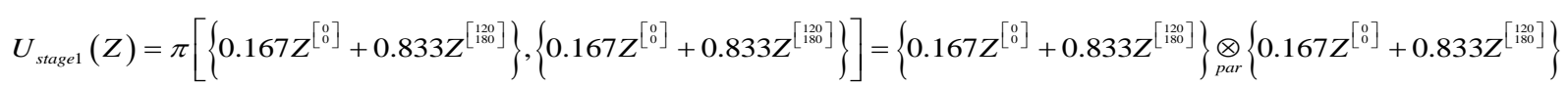

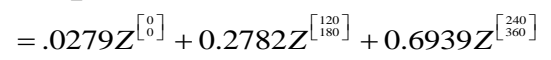

and, for stage 2, the U-function is:

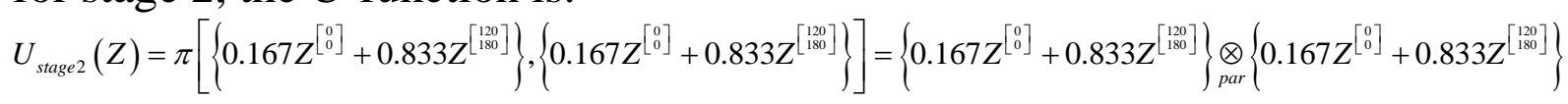

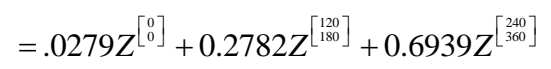


The UGF of this system configuration is obtained by applying the series $(\sigma)$ composition operators to the two serial stages as follows:

$$
\begin{aligned}
& U_{\text {system }}(Z)=\sigma\left[\left\{U_{\text {stagel }}\right\},\left\{U_{\text {stage } 2}\right\}\right]
\end{aligned}
$$

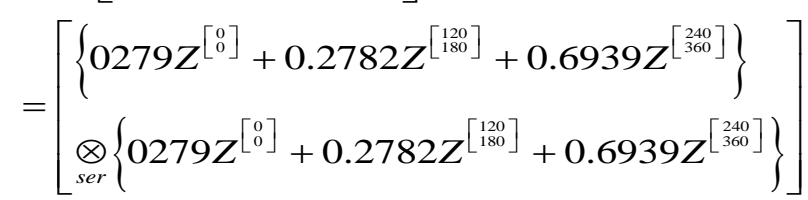

$$
\begin{aligned}
& =0.0552 Z^{[0]}+0.4634 Z^{\left[\begin{array}{l}
{[120} \\
0.00
\end{array}\right]}+0.4815 Z^{\left[\begin{array}{l}
240 \\
300
\end{array}\right]}
\end{aligned}
$$

In calculation, the UGF combine common terms and thus reduced the number of system state from eight into the above three state corresponding to the three vectors of production rates. Following the above mentioned steps, we determined the UGF function for the all the possible system configurations in figure 3 and found availability values of the different system configurations. The availability of each symmetrical configuration is presented in the figure 4 for the initial demand.

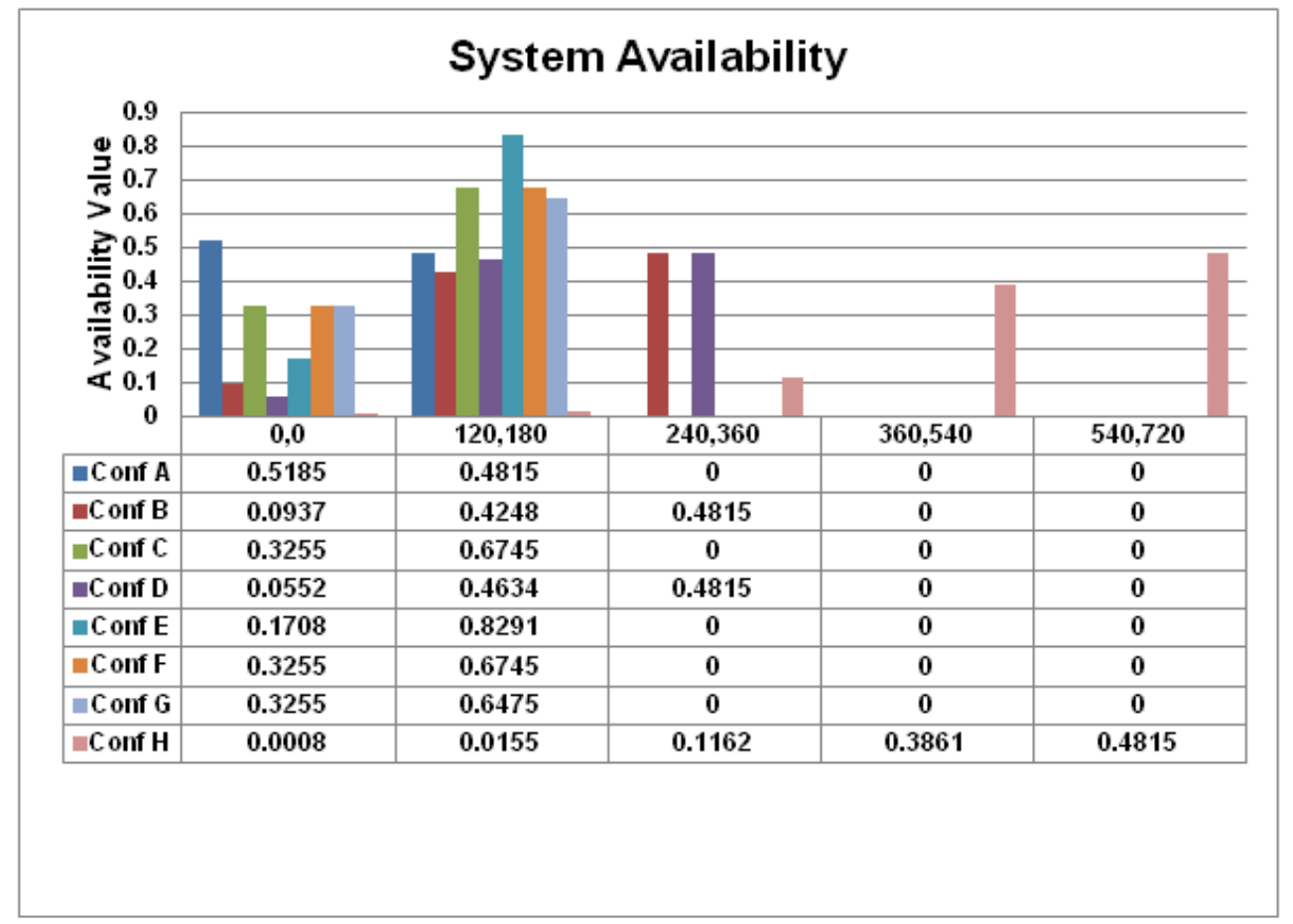

Fig. 4. Availability values for the different configurations

The expected production (EP) of the system configuration corresponding to the different part types is deduced from Eq. (14).

$$
E P=\sum_{\text {all } \mathrm{j}} R_{i} P R_{j}
$$

where EP is a vector of the expected values (expectations) of the actual production capacity (possible production rates) of the system configuration corresponding to different part types obtained using the UGF after considering availability. The feasibility of system over a period of time (that is, a configuration period) is accomplished if the expected values (expectations) of the system production capacity, corresponding to different part types, fulfill the demand 
requirements for these part types. These expectations reflect the performance (production rates) of the system in its different possible states, considering availability, and the probabilities of occurrence of these states. If the demand is 100 for part 1 and 120 for part 2 for configuration used for sample calculations, and first state in obtained UGF of configuration, does not satisfy the requirement but the remaining two states does. Hence, the system configuration availability is equal to the 0.9449 (the sum of probabilities of the satisfactory states). Similarly, if the requirements of two parts are 150 and 200 respectively, then the system configurations availability is found 0.4815 (i.e. probability associated with third state). This means that the system is $94.49 \%$ and $48.15 \%$ available to satisfy the required demand of 100,120 and 150,200 parts. Availability determination is further extended to calculate the expected production. The expected production (EP) is found 94 of part 1and 113 of part 2 using equation 14. Further, System utilization is defined as the ratio of the expected production relative to its capacity (demand). The system utilization for the sample configuration is $94 \%$ for part 1 and $94.17 \%$ for the part 2 .

Although the utilization of the system has to be below $100 \%$ to warrant its feasibility, the closer to $100 \%$ the system utilization is, the more it is able to provide the capacity needed when it is needed, which is one of the main objectives of changeable and reconfigurable manufacturing systems. Finally, High system availability reflects stability of the system in meeting its demand requirements, and system utilization reflects closeness of the system to providing the capacity needed when needed.

\section{Conclusions and Future Scope}

Availability of a manufacturing system provides a measure for its ability to meet targeted demand requirements. The use of the Universal Generating Function (UGF) technique in assessing the availability of Reconfigurable Manufacturing Systems (RMS) has been developed. One of the major contributions in the presented work is the modification of the original technique to be capable of dealing with multiple types of output performance. This allows evaluating the availability of reconfigurable manufacturing systems that produce more than one part type simultaneously. The application of the modified UGF to RMS and its computational merits in terms of reduction in the number of system states were illustrated using an example. This shows that the UGF technique is a powerful tool for comparing different systems configurations based on availability, and supporting the system designer in making the necessary tradeoffs decisions. The use of such a computationally efficient technique has an important significance in the field of reconfigurable manufacturing systems performance evaluation. It permits the evaluation of large systems in reasonable time. This application of the proposed UGF-based availability model is limited to reconfigurable manufacturing systems without buffer capacity between the different production stages. Modeling such systems with finite buffer capacity can be a valuable area for future scope. Also, one can apply this model to select the optimal configuration for multi task manufacturing system. 
Gupta, A.; Jain, P. K. \& Kumar, D.: Availability Modelling of Reconfigurable Man...

\section{References}

Aven, T. (1985). Reliability/Availability Evaluations of Coherent Systems Based on Minimal Cut Sets. Reliability Engineering, Vol., 13/2, pp. 93-104, ISSN 09518320

ElMaraghy, H.A.; Kuzgunkaya, O. \& Urbanic, R.J. (2005). Manufacturing Systems Configuration Complexity. CIRP Annals, Vol. 54/1, pp.445-450, ISSN 00078506.

Garriba, S.; Mussio, P. \& Naldi, F. (1980). Multivalued logic in the representation of engineering systems Synthesis and Analysis Methods for Safety and Reliability Studies, Apostolakis G, Garriba S, Volta G (eds.), pp.183-197, Plenum Press, NY

Griffith, W. (1980). Multistate reliability models. Journal of Applied Probability, Vol.17, pp.735-744, ISSN 00219002

Hon, K.K.B. (2005). Performance and Evaluation of Manufacturing Systems. CIRP Annals, Vol.54/2, pp.675-690, ISSN 0007-8506

Kolowrocki,K.(2000). An asymptotic approach to multistate systems reliability evaluation, Recent Advances in Reliability Theory, Methodology, Practice and Inference, pp.163-180, Birkhauser, ISBN 0-8176-4135-1, Boston

Koren,Y.;Jovane,F.;Heisel,U.;Moriwaki,T.;Pritschow,G.;Ulsoy,A.G.\&VanBrussel,H.(19 99). Reconfigurable Manufacturing Systems. CIRP Annals, Vol. 48, No. 2, pp.527-540, ISSN 0007-8506

Koren,Y.;Maler-Speredelozzi,V.\&Hu,S.J.(2003).Convertibility measure for manufacturing system. CIRP Annals- Manufacturing Technology, Vol. 52, No. 1, pp. 367-370, ISSN 0007-8506

Levitin, G. \& Lisnianski,A.(1999). Joint redundancy and Maintenance Optimization for Multistate Series- Parallel Systems. Reliability Engineering \& System Safety, Vol. 64/1, pp.33-42, ISSN 0951-8320

Lewis E.E. (1987). Introduction to Reliability Engineering, John Wiley and Sons, ISBN 0-4718-1199-8, New York

Limnios,N.\& Oprisan,G.(2001).Semi-Markov Processes and Reliability, Birkhauser, ISBN 0-8176-4198-3, Boston

Lisniansk,A.\& Levitin G.(2003). Multi-State System Reliability, World Scientific, ISBN 981-238-306-9, Singapore

Lokesh, K. \& Jain,P. K.(2012). A model and optimization approach for reconfigurable manufacturing system configuration design. International Journal of Production Research, Vol.50, No.12, ISSN 0020-7543

Murchland, J. (1975). Fundamental concepts and relations for reliability analysis of multistate systems, reliability and fault tree analysis, Theoretical and Applied Aspects of System Reliability. Society for Industrial and Applied Mathematics, Michigan

Pouret,O.; Collet,J.\& Bon, J.L.(1999). Evaluation of the unavailability of a multistatecomponent system using a binary model. Reliability Engineering and System Safety, Vol. 66 pp.13-17, ISSN 0951-8320

Ushakov, I.A. (1986). A Universal Generating Function. Soviet Journal of Computing System Science, Vol.24, pp.37-49

Vesely,W.E.; Goldberg,F.; Roberts,N.H. \& Haasl,D.F.(1981). Fault Tree Handbook, U.S. Nuclear Regulatory Commission, ISBN 0-16-005582-2, Washington 